\title{
Discusión de factores pronóstico en dos casos familiares de fiebre manchada de las Montañas Rocosas
}

\section{Discussion of prognostic factors in two familial cases of Rocky Mountain spotted fever} Jesús Delgado-de la Mora, Jesús David Licona-Enríquez, María del Carmen Candia-Plata, Gerardo Álvarez-
Hernández

\begin{abstract}
Resumen
INTRODUCCIÓN: La fiebre manchada de las Montañas Rocosas constituye un problema de salud pública en México, pues genera una elevada tasa de mortalidad. La información relacionada con factores pronóstico (estado nutricional, inmunológico e inóculo bacteriano) es escasa al respecto.

CASOS CLínICOS: Se reporta el caso clínico de dos pacientes (hermanos): un varón de 9 (caso 1) y una niña de 10 años (caso 2), procedentes de una localidad urbana del sur de Sonora, México, sin antecedentes heredofamiliares ni patológicos de relevancia para el padecimiento actual. Su dieta incluía todos los grupos alimenticios, tres veces al día. Refirieron antecedente de contacto con garrapatas, dentro y fuera de su casa, y convivencia con perros ectoparasitados. Ambos iniciaron con similar cuadro clínico (fiebre, malestar general y exantema). Se prescribió doxiciclina con el mismo esquema y día de evolución; sin embargo, uno de los pacientes falleció y el otro mejoró clínicamente, sin secuelas aparentes. De acuerdo con los hallazgos clínicos o pruebas de laboratorio se estableció el diagnóstico de fiebre manchada de las montañas rocosas. CONCLUSIONES: La diferencia en el pronóstico de los pacientes con fiebre manchada de las Montañas Rocosas puede relacionarse con el estado nutricional e inmunológico, así como con la magnitud del inóculo. Se requieren investigaciones adicionales para examinar la participación de dichos factores en el pronóstico de pacientes infectados por Rickettsia rickettsii .
\end{abstract}

PALABRAS CLAVES: Rickettsia rickettsii; enfermedades por garrapatas; pronóstico.

Abstract

BACKGROUND: Rocky Mountain spotted fever is a public health problem in Mexico, with high regional case fatality rates. Little is known regarding prognostic factors such as nutritional and immunological condition, as well as bacterial inoculum.

CLINICAL CASES: We report the cases of two siblings: a male of 9 (case 1) and a 10-yearold girl (case 2), from an urban location in southern Sonora, Mexico, with no heredofamilial or pathological history of relevance to the current condition. His diet included all the food groups, three times a day. They reported a history of contact with ticks, inside and outside their home, and living with ectoparasitized dogs with them. Both started with a similar clinical picture (fever, malaise and rash). Doxycycline was prescribed with the same scheme and day of evolution; however, one of the patients died and the other improved clinically, without apparent sequelae. According to clinical findings or laboratory tests, the diagnosis of Rocky Mountain Spotted Fever was established.

CONCLUSIONS: It is possible that the difference on the prognosis of some patients it is related to nutritional and immunological status, as well as to the magnitude of bacterial inoculum. Future research is necessary to examine the role of such factors in the prognosis of patients with Rocky Mountain spotted fever.

KEYWORDS: Rickettsia rickettsii; Tick-borne diseases; Prognosis.
Departamento de Medicina y Ciencias de la Salud, Universidad de Sonora, México.

Recibido: 1 de febrero 2017

Aceptado: 7 de marzo 2018

Correspondencia Gerardo Álvarez Hernández galvarezh63@gmail.com

Este artículo debe citarse como Delgado-de la Mora J, Licona-Enríquez JD, Candia-Plata MC, Álvarez-Hernández G. Discusión de factores pronóstico en dos casos familiares de fiebre manchada de las Montañas Rocosas. Acta Pediatr Mex. 2018;39(4):323-327. 


\section{INTRODUCCIÓN}

La fiebre manchada de las Montañas Rocosas es una enfermedad reemergente en México, provocada por Rickettsia rickettsii, que constituye un problema de salud pública. ${ }^{1,2}$ En la era pre-antibiótica (1909) se estimó una mortalidad de $65-90 \%$ en Estados Unidos ${ }^{3}$ y de $77-91 \%$ en México. ${ }^{4}$ Actualmente, los reportes del noroeste de México señalan una variación de 20-50\%, ${ }^{2,5}$ colocándola como la más severa de las Rickettsiosis del grupo de las fiebres manchadas, además de una enfermedad infecciosa con elevada mortalidad. ${ }^{6}$

Poco se sabe de los factores pronósticos de pacientes con fiebre manchada de las Montañas Rocosas, excepto por dos aspectos: 1) la relación inversa entre la administración temprana de doxiciclina (primeros 5 días de iniciar los síntomas) y 2) la posibilidad de desenlaces mortales, ${ }^{7-8}$ cuya relación puede confundirse por la participación de factores como: variedad de las cepas infectantes, condiciones climáticas, capacidad inmunológica del sujeto, entre otros. ${ }^{6,9-10}$ Incluso la deficiencia de glucosa-fosfato deshidrogenasa se asocia con un pronóstico adverso. ${ }^{11}$ Así mismo, aunque se ha discutido durante mucho tiempo, no existe certidumbre acerca de la función de factores adicionales como: estado nutricional e inmunológico de los pacientes infectados, además del tamaño del inóculo introducido. ${ }^{12,13}$

En tal escenario, con el propósito de discutir la importante función de los factores pronósticos en el resultado o consecuencia final de la enfermedad, presentamos los casos de dos hermanos con diagnóstico confirmado de fiebre manchada de las Montañas Rocosas, quienes iniciaron simultáneamente con el cuadro clínico, pero tuvieron diferente desenlace.

\section{CASOS CLÍNICOS}

En forma general, se trata de dos pacientes (hermanos): un varón de 9 (caso 1) y una niña de 10 años (caso 2), procedentes de una localidad urbana del sur de Sonora, México, sin antecedentes heredofamiliares ni patológicos de relevancia, con cartilla de vacunación completa para la edad. Su dieta incluía todos los grupos alimenticios, tres veces al día. Antecedente de contacto con garrapatas, dentro y fuera de su casa, y convivencia con perros ectoparasitados con las mismas.

En junio de 2016 iniciaron su padecimiento simultáneamente, con astenia, adinamia y fiebre no cuantificada; en su segundo día de evolución recibieron atención médica y se les indicó ampicilina por vía oral (dosis no referida), hasta el cuarto día de evolución, cuando el paciente del caso 1 cursó con mioartralgias generalizadas y lenguaje incoherente. Se efectuó una segunda revisión médica y se decidió cambiar la administración del medicamento, en ambos casos, a vía intramuscular.

Al sexto día de evolución se agregó exantema petequial generalizado en ambos pacientes, por lo que acudieron al hospital local, donde se sospechó de fiebre manchada de las montañas rocosas, decidiéndose su hospitalización. Se inició tratamiento con doxiciclina, a dosis de 2.2 $\mathrm{mg} / \mathrm{kg}$ por vía intravenosa cada 12 horas y fueron transferidos al hospital pediátrico de referencia. A continuación se describe la evolución de cada paciente, a partir de su llegada al hospital.

\section{Caso 1}

Paciente masculino de 9 años de edad, en el séptimo día de evolución, con tensión arterial de $60-40 \mathrm{mmHg}$ y frecuencia cardiaca de 60 Ipm, datos de dificultad respiratoria, edema y exantema purpúrico generalizados (Figura 1), 


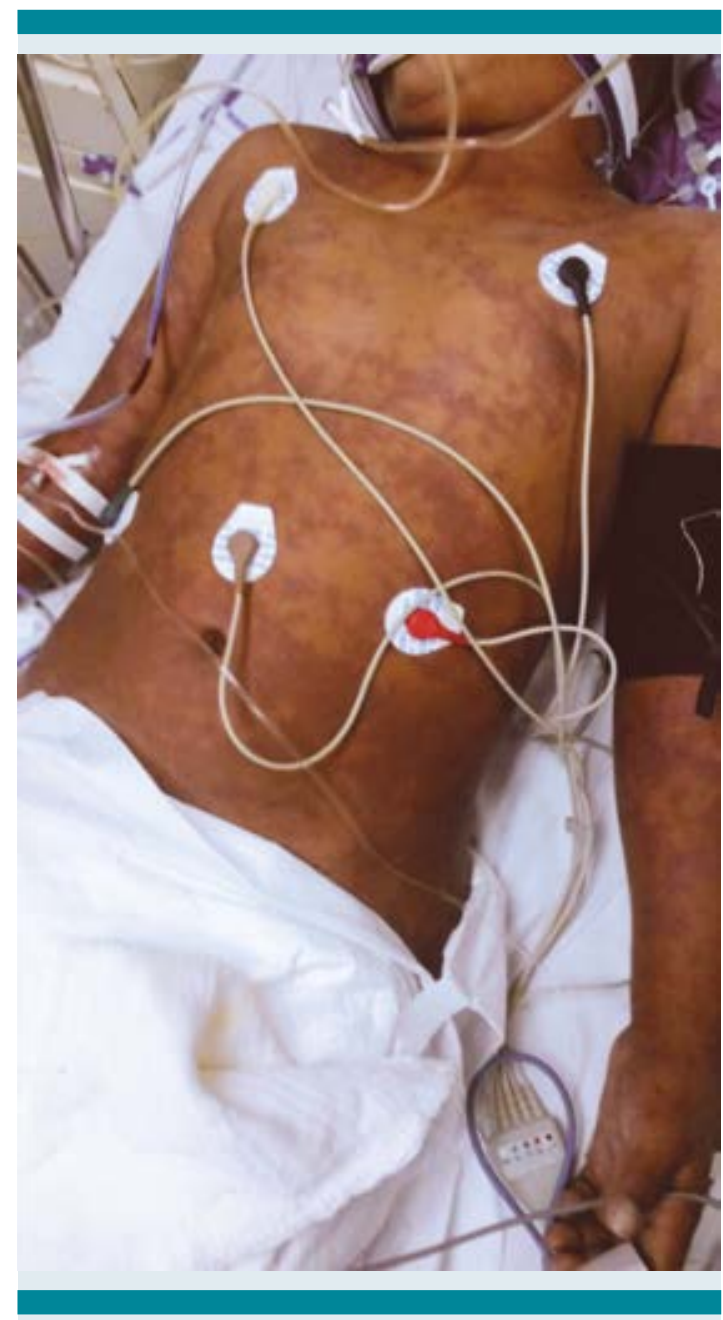

Figura 1. Caso 1. Paciente con exantema purpúrico y edema generalizado (séptimo día del ingreso hospitalario).

que afectaban las plantas y palmas. Los estudios de laboratorio reportaron leucocitosis $(13.3 \mathrm{x}$ $\left.10^{3} / \mu \mathrm{L}\right)$ con neutrofilia $\left(11.5 \times 10^{3} / \mu \mathrm{L}\right)$ y linfopenia $\left(1.02 \times 10^{3} / \mu \mathrm{L}\right)$, plaquetopenia severa $(10$ x $\left.10^{3} / \mu \mathrm{L}\right)$; concentraciones elevadas de urea $(123 \mathrm{mg} / \mathrm{dL})$ y creatinina $(1.2 \mathrm{mg} / \mathrm{dL})$; hipoalbuminemia $(1.9 \mathrm{~g} / \mathrm{dL})$, hipoglobulinemia $(1.5$ $\mathrm{g} / \mathrm{dL})$, hiponatremia (120 meq/L); elevación de glutamato-oxaloacetato transaminasa (TGO: $369.9 \mathrm{U} / \mathrm{L})$ y glutamato-piruvato transaminasa (TGP: $59.1 \mathrm{U} / \mathrm{L}$ ); bilirrubina total de $7.3 \mathrm{mg} / \mathrm{dL}$, a expensas de la directa $(5.7 \mathrm{mg} / \mathrm{dL})$, hiperlactatemia $(6.1 \mathrm{mmol} / \mathrm{L})$ y concentración alta de procalcitonina $(178.8 \mathrm{ng} / \mathrm{mL})$.

Debido a la dificultad respiratoria e insuficiencia cardiaca, se inició ventilación mecánica invasiva, se prescribieron aminas vasoactivas y se mantuvo el esquema de doxiciclina; sin embargo, a pesar del protocolo de sostén, el paciente falleció después de algunas horas de su llegada al hospital.

\section{Caso 2}

Paciente femenina de 10 años de edad, que ingresó con exantema petequial y edema generalizado (Figura 2); tensión arterial de 80-50 $\mathrm{mmHg}$ y frecuencia cardiaca de $110 \mathrm{lpm}$. Se colocó un catéter venoso central y se inició tratamiento con fármacos vasoactivos, además del mantenimiento de doxiciclina. Las pruebas de laboratorio mostraron leucocitos en los límites de referencia $\left(7.1 \times 10^{3} / \mu \mathrm{L}\right)$, con predominio de neutrófilos $\left(5.1 \times 10^{3} / \mu \mathrm{L}\right)$, linfopenia $(0.13 \times$ $\left.10^{3} / \mu \mathrm{L}\right)$ y plaquetopenia severa $\left(25 / 10^{3} / \mu \mathrm{L}\right)$; las concentraciones de urea $(26 \mathrm{mg} / \mathrm{dL})$ y creatinina $(0.4 \mathrm{mg} / \mathrm{dL})$ estuvieron dentro de los límites normales; se registró hipoproteinemia $(5 \mathrm{~g} / \mathrm{dL})$, hipoalbuminemia (2.9 g/dL), hiponatremia (129 $\mathrm{g} / \mathrm{dL})$ y elevación de procalcitonina $(42.7 \mathrm{ng} /$ $\mathrm{mL}$ ). La paciente evolucionó favorablemente,

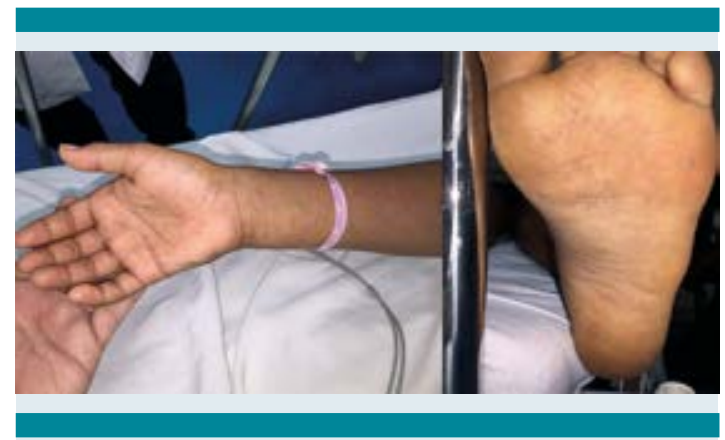

Figura 2. Caso 2. Paciente con exantema petequial generalizado, incluidas las palmas y plantas (séptimo día de hospitalización). 
sin requerir ventilación mecánica, por lo se retiró el tratamiento con aminas al tercer día de estancia hospitalaria; sin embargo, persistió con debilidad muscular generalizada e incapacidad para deambular. Al día 11 de hospitalización se otorgó el alta, por evolución satisfactoria y sin secuelas aparentes.

\section{DISCUSIÓN}

Aunque ambos casos recibieron atención médica prehospitalaria similar, tuvieron desenlace diferente, con el fallecimiento de uno y recuperación del otro.

Si bien la fiebre manchada de las Montañas Rocosas se ha descrito como una enfermedad con elevada motalidad, ${ }^{2-4}$ uno de sus mecanismos de acción relacionado con mal pronóstico tiene que ver con la deficiencia de glucosa6-fosfato deshidrogenasa, que disminuye la capacidad oxidativa del eritrocito y genera diversas complicaciones clínicas. ${ }^{11}$

La administración de doxiciclina en los primeros cinco días de iniciar los síntomas se asocia con mejor pronóstico para el paciente, ${ }^{7-8}$ incluso se ha observado disminución de la tasa de mortalidad a 1\% en Estados Unidos. ${ }^{1}$ En Sonora ocurre un panorama distinto, pues recientemente se ha reportado una tasa de mortalidad superior a $20 \%$, a pesar del tratamiento oportuno. ${ }^{2,5}$ Esta variación puede relacionarse con la clasificación imprecisa de los casos de rickettsiosis del grupo de las fiebres manchadas, que son inapropiadamente diagnosticados como fiebre manchada de las montañas rocosas. Tal situación se ha observado, también, en regiones de Estados Unidos, donde reportan la detección de otras especies de Rickettsia del grupo de las fiebres manchadas como: R. montana, R. amblyommii y R. parkeri, mismas que generan un cuadro clínico menos severo y de mejor pronóstico que la variante específica de la fiebre manchada de las Montañas
Rocosas. ${ }^{13-14}$ La inadecuada clasificación puede obedecer a reacción cruzada de $R$. rickettsii con otras especies de Rickettsia o a la detección de anticuerpos inducidos por infecciones rickettsiales previas, lo que puede ocurrir cuando se considera como caso confirmado en un paciente con prueba única de inmunofluorescencia indirecta y subsiguientemente reportar resultados falsos positivos en las pruebas serológicas. ${ }^{6}$

Ante esta situación, se requiere explorar factores adicionales, potencialmente relacionados con el pronóstico de los pacientes con fiebre manchada de las Montañas Rocosas; por ejemplo, ensayos in vivo e in vitro reportan que un mayor inóculo de $R$. rickettsii reduce el tiempo de incubación e incrementa la tasa de infección, referida como la manifestación de signos y síntomas sugerentes de la enfermedad. ${ }^{12,15}$ Por lo tanto, es razonable suponer que las diferencias en la cantidad del inóculo se asocien con un resultado clínico distinto, como sucedió en los pacientes expuestos en este estudio. Así, es conveniente que investigaciones futuras exploren esta probable relación.

Aunque es conocida la función del estado inmunológico y nutricional en la patogénesis de las enfermedades infecciosas, ${ }^{16,17}$ se ha evaluado muy poco acerca del pronóstico de pacientes con fiebre manchada de las Montañas Rocosas. De acuerdo con la relevancia clínica de la enfermedad en la población pediátrica de México, ${ }^{18}$ misma que es particularmente vulnerable a esta enfermedad, se requieren investigaciones del efecto inmunológico y deficiencias nutricionales en el pronóstico de los pacientes infectados por R. rickettsii.

\section{REFERENCIAS}

1. Biggs HM, Barton C, Bradley KK, Dahlgren FS, Drexler NA, Dumler JS, et al. Diagnosis and management of tick borne rickettsial diseases: Rocky Mountain spotted fever 
and other spotted fever group rickettsiosis, ehrlichiosis and anaplasmosis - United States. MMWR Recomm Rep 2016;65(2):1-44.

2. Delgado-de la Mora J, Licona-Enríquez JD, Leyva-Gastélum M, Delgado-de la Mora D, Rascón-Alcantar A, ÁlvarezHernández G. Una serie fatal de casos de fiebre manchada de las Montañas Rocosas en Sonora, México. Biomédica 2018;38(1). DOI: http://dx.doi.org/10.7705/biomedica. v38i0.3507

3. Ricketts HT. Some aspects of Rocky Mountain spotted fever as shown by recent investigations. 1909. Rev Infect Dis 1991;13(6):1227-40.

4. Bustamante ME, Varela G. Características de la fiebre manchada de las Montañas Rocosas en Sonora y Sinaloa, México. Rev Inst Salub Enf Trop1944;5:129-136.

5. Alvarez-Hernandez G, Murillo-Benitez C, Candia-Plata MC, Moro M. Clinical profile and predictors of fatal Rocky Mountain spotted fever in children from Sonora, Mexico. Pediatr Infect Dis J 2015;34(2):125-30.

6. Raoult D, Parola P. Rocky mountain spotted fever in the USA: a benign disease or a common diagnostic error? Lancet Infect Dis 2008;8(10):587-9.

7. Kirkland KB, Wilkinson WE, Sexton DJ. Therapeutic delay and mortality in cases of Rocky Mountain spotted fever. Clin Infect Dis 1995;20(5):1118-21.

8. Dalton MJ, Clarke MJ, Holman RC, Krebs JW, Fishbein DB, Olson JG, et al. National surveillance for rocky mountain spotted fever, 1981-1992: epidemiologic summary and evaluation of risk factors for fatal outcome. Am J Trop Med Hyg 1995;52(5):405-13.

9. Parola P, Socolovschi C, Jeanjean L, Bitam I, Fournier PE, Sotto $A$, et al. Warmer weather linked to tick attack and emergence of severe Rickettsioses. PLoS Negl Trop Dis 2008;2(11):e338.
10. Clark TR, Noriea NF, Bublitz DC, Ellison DW, Martens C, Lutter El, et al. Comparative genome sequencing of Rickettsia rickettsii strains that differ in virulence. Infect Immun 2015;83(4):1568-76.

11. Walker DH, Hawkins HK, Hudson P. Fulminant Rocky Mountain spotted fever. Its pathologic characteristics associated with glucose-6-phosphate dehydrogenase deficiency. Arch Pathol Lab Med 1983;107(3):121-5.

12. DuPont HL, Hornick RB, Dawkins AT, Heiner GG, Fabrikant IB, Wisseman CL, et al. Rocky Mountain spotted fever: a comparative study of the active immunity induced by inactivated and viable pathogenic Rickettsia rickettsii. J Infect Dis 1973;128:340-44.

13. Raoult D, Paddock CD. Rickettsia parkeri infection and other spotted fever in the United States. N Engl J Med 2005;353(6):626-27.

14. Secretaría de Salud. Prevención, diagnóstico y tratamiento de la fiebre manchada por Rickettsia rickettsii en población pediátrica y adulta, en el primer y segundo nivel de atención. México. 2013. [en línea]. Dirección URL: <http://www. cenetec.salud.gob.mx/interior/gpc.html>.

15. Moncayo AC, Cohen SB, Fritzen CM, Huang E, Yabsley MJ, Freye JD, et al. Absence of Rickettsia rickettsii and occurrence of other spotted fever group Rickettsioses in ticks from Tennessee. Am J Trop Med Hyg 2010;83(3):653-57.

16. Woods ME, Olano JP. Host defenses to Rickettsia rickettsii infection contribute to increased microvascular permeability in human cerebral endothelial cells. J Clin Immunol 2008;28(2):174-85.

17. Medzhitov R. Recognition of microorganisms and activation of the immune response. Nature 2007;449(7164):819-26.

18. Katona $P$, Katona-Apte J. The interaction between nutrition and infection. Clin Infect Dis 2008;46(10):1582-88.

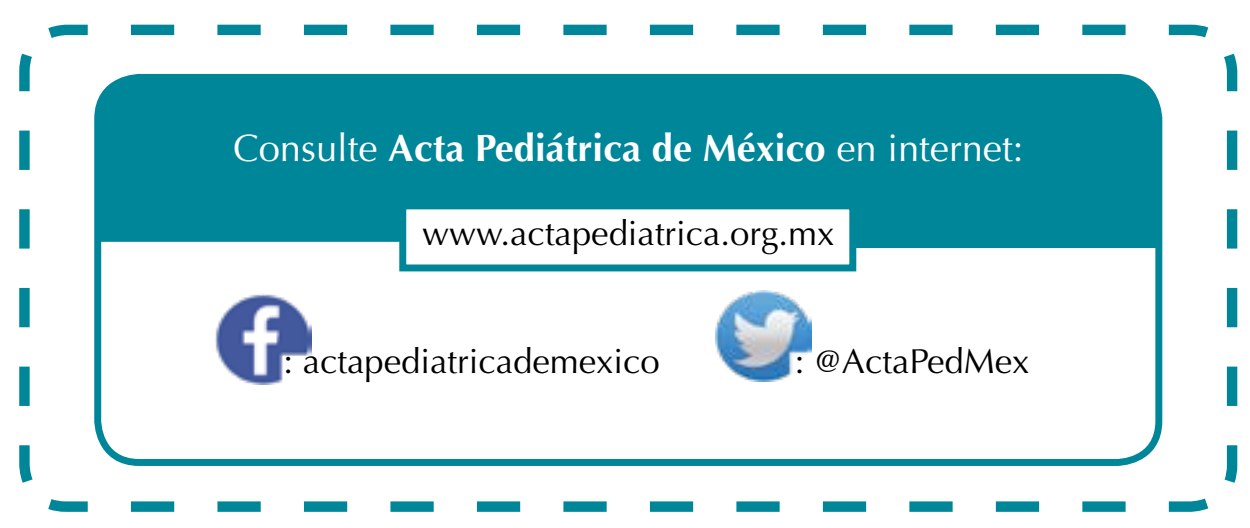

